\title{
Microstructure Modelling of Hot Deformation of Al-1\%Mg Alloy
}

\author{
J. Talamantes-Silva ${ }^{1}$, M.F. Abbod ${ }^{2}$, E.S. Puchi Cabrera ${ }^{3}$, \\ I.C. Howard, J.H. Beynon ${ }^{4}$, C.M. Sellars and D.A. Linkens \\ IMMPETUS \\ Institute for Microstructural and Mechanical Process Engineering: The University of Sheffield, \\ Mappin Street, Sheffield S1 3JD, UK \\ Now at \\ ${ }^{1}$ Sheffield Forgemasters Engineering Ltd, Brightside Lane, Sheffield, S9 2RW, UK \\ ${ }^{2}$ School of Engineering and Design, Brunel University, Uxbridge UB8 3PH, UK \\ ${ }^{3}$ Facultad de Ingenieria, Universidad Central de Venezuela, Caracas, Venezuela \\ ${ }^{4}$ Faculty of Engineering \& Industrial Sciences, Swinburne University of Technology, Hawthorn, VIC 3122, \\ Australia
}

\begin{abstract}
This study presents the application of the finite element method and intelligent systems techniques to the prediction of microstructural mapping for aluminium alloys. Here, the material within each finite element is defined using a hybrid model. The hybrid model is based on neuro-fuzzy and physically based components and it has been combined with the finite element technique. The model simulates the evolution of the internal state variables (i.e. dislocation density, subgrain size and subgrain boundary misorientation) and their effect on the recrystallisation behaviour of the stock. This paper presents the theory behind the model development, the integration between the numerical techniques, and the application of the technique to a hot rolling operation using aluminium, 1wt $\%$ magnesium alloy. Furthermore, experimental data from plane strain compression (PSC) tests and rolling are used to validate the modelling outcome. The results show that the recrystallisation kinetics agree well with the experimental results for different annealing times. This hybrid approach has proved to be more accurate than conventional methods using empirical equations.
\end{abstract}

Keywords: modelling, finite element, hybrid model, hot rolling, neuro-fuzzy, microstructure, recrystallisation. 


\section{Introduction}

Modelling the microstructural evolution in aluminium alloys during hot deformation, in which static recrystallisation plays a major role, has proved to be a difficult task. Different physically-based models have been developed in which recrystallisation is analysed in terms of nucleation and growth. The internal state variables used in these phenomenological models are the main factors that control the flow stress and recrystallisation kinetics of the material. Thus, suitable prediction of these variables is essential. During hot deformation operations such as forging, rolling and extrusion, the workpiece undergoes continuous changes in the strain rate and temperature during straining. Such changes have a significant effect on the evolution of the microstructure and subsequent recrystallisation behaviour [1][2].

This paper shows how the combination of a hybrid model with the finite element (FE) technique can be used to develop a model for predicting the evolution of the internal state variables (i.e. dislocation density, subgrain size and subgrain boundary misorientation) and subsequent recrystallisation behaviour. This hybrid model has been developed for aluminium alloys and it covers a wide range of deformation conditions, namely temperature, strain rate and time. The model is built to include compositional ranges, but this paper presents results for only one alloy composition. The model predicts the evolution of the transient transformation behaviour of the internal states using neuro-fuzzy models and the final flow stress using phenomenological equations based on internal state variables. Subsequently, the static recrystallisation kinetics have also been predicted using physically-based equations.

Hybrid modelling techniques provide a valuable modelling tool combining different modelling approaches in one dynamic model [3]. The hybrid model efficiently combines black-box data-driven models (neuro-fuzzy) and white-box models (physical equations). The white-box model ensures that the results are physically acceptable, while the black-box model is used to increase the accuracy of the results. A hybrid modelling approach was adopted in this study using parallel and serial parametric and non-parametric models. They have the advantages of dealing better with sparse data, extrapolation and uncertainty in the initial model [4].

It is common practice to use plane strain compression (PSC) testing to establish the flow stress behaviour of a particular material [5] under different hot working conditions. The derived constitutive empirical relationship is then used in computer models to simulate thermomechanical processes such as rolling, forging or extrusion.

The finite element method is a well established and increasingly popular technique for such thermomechanical simulations. When using this technique, the local prediction of strain, strain rate and temperature can be mapped and then used in other models to predict microstructural evolution. This strategy was adopted in the present work to predict the microstructural evolution and recrystallisation kinetics of aluminium alloys using phenomenological models. Furthermore, a comparison of finite element results between classical constitutive equations and the embedded neuro-fuzzy approach developed in this work is presented. This is done using both hot rolling and PSC testing of an Al-1wt\% magnesium alloy (Al-1\%Mg). 


\section{Physical Modelling of Materials Properties}

Modelling of the thermomechanical processing effects on metal properties during rolling, forging and extrusion involves local regions undergoing change of deformation conditions. Such changes involve a continuous change in strain rate and/or temperature and changes in strain path. A decrease in strain rate and change in straining direction have been found to have a significant effect on the evolution of deformation microstructure, texture and subsequent recrystallisation behaviour [6][7][8][9].

\subsection{Microstructure Internal States}

The reason for the above effects is considered to arise from the different evolution of so-called geometrically necessary dislocations during monotonic and reversed deformation, or during change in strain rates. The dislocation density within sub-boundaries, $\rho_{b}$, changes according to the relationship $\rho_{b}=C \theta / b \delta$, where $\mathrm{C}$ is a geometrical constant $(\approx 1-2$ depending on the type of the sub-boundaries), $\theta$ is the subboundary misorientation in radians, $\mathrm{b}$ is the Burger vector, and $\delta$ is the mean linear intercept subgrain size. The value of $\mathrm{C}$ increases with strain in monotonic deformation, but decreases with strain after change in straining direction [6][7][8][9] or decrease in strain-rate [7][10][11]. On the other hand, spacing between the sub-boundaries $\delta$ decreases while misorientation across the sub-boundaries $\theta$ increases.

The evolution of dislocation substructure and subsequent recrystallisation behaviour under deformation conditions with changes in strain-rate or strain path is very complex. The complex evolution of dislocation substructure cannot be quantitatively described using conventional evolution laws [12][1], which have been developed based on experimental results obtained in laboratory scale tests under constant deformation conditions. By considering the internal states produced by deformation, substructure and subsequent recrystallisation behaviour can be modelled successfully for a commercial $\mathrm{Al}-1 \% \mathrm{Mg}$ alloy [13].

\subsection{Modelling of Flow Stress}

Modelling of the flow stress is carried out in terms of the internal state variables $\left(\rho_{\mathrm{i}}^{-1 / 2}, \delta, \theta\right)$ where $\rho_{\mathrm{i}}$ is the dislocation density inside the subgrains, $\delta$ and $\theta$ are the subgrain boundary parameters defined above. These in turn are determined by the deformation conditions of temperature, strain rate and strain $(\mathrm{T}, \dot{\varepsilon}, \varepsilon)$. The final flow stress $(\sigma)$ is the sum of the effective stress $\left(\sigma_{\mathrm{e}}\right)$ (known as friction stress $\left(\sigma_{\mathrm{f}}\right)$ [7] and the internal stress $\left(\sigma_{\mathrm{i}}\right)$ that arises from microstructure as shown in equation 1.

$$
\begin{aligned}
\sigma & =\sigma_{e}+\sigma_{i} \\
\sigma_{i} & =\sigma_{\rho i}+\sigma_{\delta}+\sigma_{d}+\sigma_{p}
\end{aligned}
$$

where $\sigma_{\rho \mathrm{pi}}$ is the thermal stress due to interaction of dislocations inside subgrains, $\sigma_{\delta}$ is the long range internal back stress due to subgrain boundaries, $\sigma_{\mathrm{d}}$ is the stress arising from grain boundaries, $\sigma_{\mathrm{p}}$ is the stress due to second phase particles. 
In single phase polycrystals with large grains compared to subgrains, which undergo only recovery processes such as for aluminium alloys, the terms $\sigma_{\mathrm{p}}$ and $\sigma_{\mathrm{d}}$ can be neglected and the internal stress is mainly the sum of $\sigma_{\rho i}$ and $\sigma_{\delta}$. Therefore the final flow stress during deformation involves stresses arising from internal dislocation $\left(\sigma_{\rho \mathrm{i}}\right)$ from subgrain boundaries $\left(\sigma_{\delta}\right)$ and the friction stress arising from glide of internal dislocations $\left(\sigma_{\mathrm{f}}\right)$ as $[1]$ :

$$
\begin{gathered}
\sigma_{\rho i}=\alpha_{1} M G b \rho_{i}^{1 / 2} \\
\sigma_{\delta}=\alpha_{2} M G b / \delta \\
\sigma_{f}=\frac{M B}{D_{0} b^{2}} \cdot \frac{Z}{\rho_{m}}
\end{gathered}
$$

where $\alpha_{1}=0.38$ and $\alpha_{2}=0.79$ for aluminium alloys are constants, $M=3$ for polycrystals is the Taylor factor, $\mathrm{D}_{0}=1 \mathrm{~m}^{2} / \mathrm{s}$ is the diffusion frequency, $\mathrm{G}=(29484-13.6 \mathrm{~T}) \mathrm{MPa}$ is the shear modulus, $b=0.286 \times 10^{-9} \mathrm{~m}$ is the Burgers vector, $\rho_{\mathrm{m}}$ is the mobile dislocation density, which is the same order of magnitude as internal dislocation density $\rho_{\mathrm{i}}$ for hot deformation at constant strain rate, $\mathrm{B}$ is a material constant reflecting solution hardening, and $\mathrm{Z}$ is the Zener-Hollomon parameter.

\subsection{Modelling of Recrystallisation Behaviour}

Nucleation of recrystallisation takes place in different places within a deformed material. A generalised model to relate the internal state variables and their distribution has been proposed [13] and is described by equations (5-11). The number of nuclei formed per unit volume,

$$
N_{V}=p_{1} \lambda_{1} P_{V}+p_{2} \lambda_{2} \frac{L_{V}}{\delta}+p_{3} \lambda_{3} \frac{S_{V}}{\delta^{2}}+p_{4} \lambda_{4}+\frac{P_{\theta}}{\delta^{3}}
$$

where $P_{V}$ is the number of grain corners per unit volume, and $L_{v}$ is the line length per unit volume at which three grains meet, $S_{v}$ is the grains boundary surface area per unit volume, $P_{\theta}$ is the probability of finding mobile sub-boundaries, $\lambda_{1}-\lambda_{4}$ are geometrical parameters and $\mathrm{p}_{1}-\mathrm{p}_{4}$ are probabilities for finding subgrains with a size larger than a critical value that can provide the nuclei for recrystallisation related to the four different sites. For plane strain compression tests (PSC),

$L_{v}=1.513\left[\exp (\varepsilon / 1.155)+0.5(1+\exp (-(\varepsilon / 1.155))] \cdot d_{0}^{-2}\right.$

$S_{v}=\left(0.429 \exp (-\varepsilon / 1.155)+\exp ((\varepsilon / 1.155)+0.571) \cdot d_{0}^{-1}\right.$

where $\mathrm{d}_{0}$ is the original grain size.

As the strain regime covers a wide range of thermomechanical processing conditions and the grain boundaries surfaces are the most significant places for nucleation, the equation can be simplified to:

$$
N_{V} \approx p_{3} \lambda_{3} \frac{S_{v}}{\delta^{2}}
$$

where for the present alloy $d_{0}=117 \mu \mathrm{m}$ and $p_{3}=0.0191$. 
Recrystallisation kinetics is determined by both nucleation density and growth rate of nuclei. If the nucleation is site-saturated, which is a reasonable approximation after hot deformation, then the following kinetics law of recrystallisation is obtained:

$$
X(t)=1-\exp \left(-X_{e x t}(t)\right)
$$

where $X(t)$ is the fraction recrystallised after annealing time $t$ and $X_{\text {ext }}(t)$ is the corresponding extended volume which is determined by:

$$
X_{e x t}(t)=\frac{3}{4} \pi N_{V}\left(G_{r} \cdot t\right)^{3}
$$

where $G_{r}$ is the growth rate of the recrystallisation nuclei which is mainly affected by the recovery from the

deformed microstructure, and by the spatial distribution of the stored energy on the scale of the grain size, which is the same as that calculated from experimental results of the internal dislocation structure and statistical analysis and is related to the stored energy $\left(P_{D}, \mathrm{~J} \cdot \mathrm{m}^{-3}\right)$ and calculated by:

$$
P_{D}=\frac{G b^{2}}{10}\left[\rho_{i}\left(1-\ln \left(10 b \rho_{i}^{1 / 2}\right)\right)+\frac{2 \theta}{b \delta}\left(1+\ln \left(\frac{\theta_{c}}{\theta}\right)\right)\right]
$$

where $\theta_{c}$ is the critical angle for distinguishing between a grain and subgrain boundary (approximately $15^{\circ}$ ).

The time for $50 \%$ recrystallisation can be calculated by the following equation

$$
t_{50}=C_{3} P_{D}^{-1} N_{V}^{-1 / 3}
$$

where $\mathrm{C}_{3}$ is a temperature-dependent material constant. For the present alloy at $400^{\circ} \mathrm{C}\left(C_{3}=7 \times 10^{10} \mathrm{~J} . \mathrm{s} . \mathrm{m}^{-4}\right)$.

For site-saturated nucleation, the recrystallised grain size is simply calculated from the nucleation density as:

$$
d_{\text {rex }}=A N_{v}^{-1 / 3}
$$

where $A$ is a geometric parameter to relate the surface linear intercept size and spatial diameter of the grains. For a grain structure of tetrakiadecahedra $A=0.2347$.

\section{Hybrid Modelling Developments}

The combination of physical knowledge and black-box data, termed "hybrid model" in this work, is an attractive way for further development of modelling. It has been shown [4][14] that hybrid modelling approaches can be applied to simulate plastic deformation behaviour and microstructural evolution during thermomechanical processing.

\subsection{Neuro-Fuzzy Modelling}

Intelligent systems researchers have developed computational theories that begin with well-defined computational goals and that provide information-processing descriptions of target behaviours in terms of inputs, outputs, and algorithms [15].

Modelling plays a very important role in designing an intelligent system, because in modelling one tries to uncover what actually happens in the natural system. Not only should a model reproduce some features of the 
natural system it is supposed to describe, but its formulation should also be consistent with what is known about the considered natural system: parameters cannot take arbitrary values, and the mechanisms and structures of the model must have some physical plausibility. Furthermore, the model should make testable predictions, and ideally all variables and parameters should be accessible to experiment.

The Adaptive Network based Fuzzy Inference System (ANFIS) architecture and learning is based on a fuzzy inference system [16] implemented in a framework of an adaptive network. Using a hybrid learning procedure, ANFIS can learn an input-output mapping based on human knowledge (in the form of if-then fuzzy rules). The ANFIS architecture has been employed to model non-linear functions, identify non-linear components on-line in a control system, and predict a chaotic time series. ANFIS performs the identification of an input-output mapping, available in the form of a set of $\mathrm{N}$ input-output examples, with a fuzzy architecture, inspired by the Takagi-Sugeno modelling approach [17].

\subsection{Internal States Evolution}

The dynamic evolution of the internal states has been modelled using a neuro-fuzzy technique which has been applied to hot deformation of $\mathrm{Al}-1 \% \mathrm{Mg}$ alloy during plane strain compression tests with constant, increasing and decreasing strain rates.

Based on the developed model for the stress-strain relationship the steady-state levels of the internal states are calculated and utilised to scale the dynamic behaviour of each state for the selected composition with respect to the temperature and strain rate. The internal states are the dislocation density (geometrically-necessary and random), the subgrain size and misorientation. The evolution equation for the internal variables can be represented by the following equation:

$$
\dot{S}_{j}=f\left(T, \dot{\varepsilon}, S_{1}, \ldots, S_{n}\right)
$$

where $S_{j}$ is a microstructural characteristic.

\subsection{Hybrid Model}

After the internal states have been calculated, the stress due to each individual state can be calculated and summed together to calculate the final stress. Nevertheless, there is the friction stress which is another component to be added to the final stress. The friction stress is not measurable and therefore it is estimated as the difference between the final stress (measured) and the summed stress due to the internal states. This approach means that the stress/strain data used in the FE models of deformation processes are accurate, and only the internal states captured in the microstructure are used to compute the recrystallisation behaviour. This distinction is hidden in the empirical modelling.

In the hybrid model, (Figure 1), the model inputs are the composition, temperature, strain-rate and strain. The model calculates the internal state variables, the final flow stress and the recrystallisation behaviour $\left(\mathrm{N}_{\mathrm{v}}, \mathrm{d}_{\mathrm{rex}}\right.$, $t_{50}$ ). Since the model covers a wide range of temperature and strain rate for each individual composition, it 
was important to tune the model to adjust some of the parameters for scaling the internal state variables and calculating the individual internal stresses.

\section{Conventional Empirical Modelling}

The conventional empirical model is based on the following equations for calculating the stress/strain $(\sigma-\varepsilon)$ relationships:

$$
\sigma=\sigma_{0.05}+\left(\sigma_{s s}-\sigma_{0.05}\right)\left[1-\exp \left(\frac{-\varepsilon}{\varepsilon_{r}}\right)\right]^{0.5}
$$

where $\sigma_{0.5}$ is the stress at a strain of $0.05, \sigma_{\mathrm{ss}}$ is the steady state stress, $\varepsilon_{\mathrm{r}}$ is the strain at which

$$
\left(\frac{\sigma-\sigma_{0.05}}{\sigma_{s s}-\sigma_{0.05}}\right)=0.795 \text { and }
$$

$$
\varepsilon_{r}=a+b \sigma_{s s}^{2}
$$

where $\mathrm{a}$ and $\mathrm{b}$ are composition dependant constants with values of 0.074 and $2.47 \times 10^{-5}$ respectively for the present alloy and

$$
\begin{aligned}
& \sigma_{s s}=45.4545 \sinh ^{-1}\left(\left(\frac{Z}{196 \times 10^{9}}\right)^{1 / 6.0}\right) \\
& \sigma_{0.05}=25.905 \sinh ^{-1}\left(\left(\frac{Z}{19 \times 10^{9}}\right)^{1 / 6.7}\right)
\end{aligned}
$$

$Z$ is the Zener-Hollomon parameter, which is defined by:

$$
Z=\dot{\varepsilon} \exp \left(\frac{Q_{d e f}}{R T}\right)
$$

where $\dot{\varepsilon}$ is the equivalent strain rate, $Q_{d e f}$ is the activation energy for deformation $\left(Q_{d e f}=156 \mathrm{~kJ} / \mathrm{mol}\right)$, and $T$ is the deformation temperature measured in Kelvin.

The relationship between the fraction recrystallised $\left(X_{V}\right)$ and the holding time $(t)$ is generally represented by the following equation:

$$
X_{v}=1-\exp \left[-0.693\left(\frac{t}{t_{0.5}}\right)^{k}\right]
$$

where $t$ is annealing time, $k$ is the Avrami exponent with a commonly reported value of 2 [18], and $t_{50}$ is the time to $50 \%$ recrystallisation. For the calculation of $t_{50}$, the empirical model bypasses the evolution of substructure, and relates the final microstructure to strain, strain rate and temperature by regression of the experimental data.

$$
t_{0.5}=\alpha 10^{-3} Z^{-1.1} \varepsilon^{-2.7} \exp \left(\frac{Q_{r e x}}{R T}\right)
$$


where $\varepsilon$ is the final equivalent strain, $Q_{\text {rex }}$ is the activation energy for recrystallisation $\left(Q_{\text {rex }}=230 \mathrm{~kJ} / \mathrm{mol}\right), R$ is the universal gas constant $\left(R=8.31 \mathrm{~J} \cdot \mathrm{mol}^{-1} \cdot \mathrm{K}^{-1}\right)$, and $T$ is the annealing temperature measured in Kelvin and , and $\alpha$ is a constant ( $\alpha=5.59$ for the present alloy). The recrystallised grain size is calculated by

$$
d_{r e x}=1.81 \times 10^{5} Z^{-0.24} \varepsilon^{-0.39}
$$

\section{Finite Element Modelling}

The finite element method has been used to simulate both hot rolling and PSC testing of Al-1\% Mg. The first model focuses on hot rolling on a laboratory mill. Figure 2 shows a schematic of the hot rolling process including the thermal phenomena involved. Here, the hot aluminium alloy (at about $420^{\circ} \mathrm{C}$ ) is drawn into the roll gap, where its thickness is reduced by up to $50 \%$. The process was simulated using a two-dimensional finite element model (plane strain), which was built using a commercial general purpose finite element software package (MSC.MARC). Figure 3(a) shows the finite element mesh used for the analysis. The stock was modelled as a rigid viscoplastic material, $100 \mathrm{~mm}$ long with a thickness of $20 \mathrm{~mm}$ using 2-dimensional quadrilateral elements, which are full integration 4-node plane strain elements. This element uses 4 integration or Gauss points to evaluate the behaviour inside every element. Symmetry conditions were applied at the bottom of the stock; thus only half of the process was modelled. The model was thermomechanically coupled and all the material properties were functions of temperature. The heat transfer coefficient at the contact zone was assumed to be $50 \mathrm{~kW} \cdot \mathrm{m}^{-2} \cdot \mathrm{K}^{-1}$. The heat generated by both friction and plastic work was included, with 0.95 of the plastic work converted to heat.

To represent friction an algorithm based on Coulomb's law was applied:

$$
f_{t}=\mu f_{n}
$$

where $\mu$ is the coefficient of friction, $f_{t}$ is the tangential or friction force and $f_{n}$ is the normal reaction. For the finite elements models the above equation was modified thus:

$$
f_{t}=\mu f_{n} \frac{2}{\pi} \arctan \left(\frac{v_{r}}{C}\right)
$$

where $v_{r}$ is the relative sliding velocity and $C$ is a constant that has a typical value of $10 \%$ of the mean sliding velocity. This formula smoothes the sudden changes in direction of the frictional forces at the neutral zone.

The stock was modelled as an $\mathrm{Al}-1$ weight $\%$ magnesium alloy $(\mathrm{Al}-1 \% \mathrm{Mg})$ and its flow stress was represented using two different approaches: a conventional constitutive equation [19] and the hybrid model developed in this work as described in the preceding sections of the paper. For both, the flow stress depends upon the strain, strain rate, and temperature.

The model was thermomechanically coupled, incorporating thermal effects on the deformation behaviour of the stock (see Figure 2). The heat generated by friction at the roll-stock interface was evaluated as: 


$$
Q_{f}=f_{f} \cdot v_{r} \cdot Q_{m}
$$

where $Q_{f}$ is the heat generated by friction, $f_{f}$ is the friction force, $v_{r}$ is the relative sliding velocity and $Q_{m}$ is the mechanical equivalent of heat. In addition, the heat generated by plastic deformation was considered in the modelling.

For the PSC test, the specimen was again modelled as a rigid viscoplastic material, 50mm long with a thickness of $10 \mathrm{~mm}$, and the tools were modelled as rigid, with heat transfer. The initial temperature was set to $400^{\circ} \mathrm{C}$ and the nominal strain rate was $1 \mathrm{~s}^{-1}$. Symmetry conditions were applied at the bottom and on the right side; thus only one quarter of the specimen was modelled as shown in Figure $3 b$. The same type of element and thermal boundary condition as in the rolling case were applied. Automatic re-meshing was used during this analysis to avoid excessive element distortion and to produce a finer mesh.

\section{Experimental Procedure}

The experimental procedure used for PSC testing and measurement of fraction recrystallised after subsequent annealing has been described in detail elsewhere [20][18][21]. For the specific conditions in this paper, tests were carried out on a laboratory melted $\mathrm{Al}-1 \% \mathrm{Mg}$ alloy, designated Mat $\mathrm{B}$ previously [21], of composition, by weight: $1.06 \% \mathrm{Mg}, 0.12 \% \mathrm{Si}, 0.47 \% \mathrm{Fe}, 0.016 \% \mathrm{Cu}, 0.005 \% \mathrm{Mn}, 0.013 \% \mathrm{Ti}, 0.02 \% \mathrm{Zn}$, remainder $\mathrm{Al}$, and initial grain size of $90 \mu \mathrm{m}$. The test furnace temperature was $400^{\circ} \mathrm{C}$, and the tools were heated so that the faces in contact with the specimen were also at $400^{\circ} \mathrm{C}$. The specimens of initial thickness $10 \mathrm{~mm}$ were deformed at an equivalent strain rate of $1 \mathrm{~s}^{-1}$ to an equivalent strain of 0.67 . They were then held in the test furnace for times of 40,100, 160 and $320 \mathrm{~s}$ and water quenched to room temperature.

A central longitudinal section through the deformed region was mechanically polished, electropolished and anodised for measurement of the fraction recrystallised. Measurements were made under polarised light using a graticule with 21 points in the eyepiece of the microscope. The fraction of the 21 points falling in the recrystallised grains was counted. The magnification was such that no two adjacent points fell in the same grain. After each count, the specimen was moved so that the adjacent area on the traverse line was measured. Point counting was carried out on 16 traverse lines through the thickness and $1 \mathrm{~mm}$ apart, so that the distribution of recrystallisation across the whole of the deformed area was determined. Values in the four quadrants were averaged to eliminate any asymmetry, and the resulting distributions are presented as contours on the quadrant with the left edge in line with the edge of the tool and the bottom right hand corner at the centre of the specimen.

The hybrid model and the training data used in this work have been reported elsewhere [22][23]. For the conventional approach, the model was based on equations (13-20). For comparison of the overall recrystallisation kinetics from experiment with those from the models, the mean value of fraction recrystallised in the deformation zone was determined from the contour plots for local fraction recrystallised by linear summation of the area fractions of the different values of $X$. 


\section{Results and Discussion}

Figure 4 (a), (b) and (c) show the microstructure variables computed by the hybrid model as a function of strain, and Figure 4 (d) shows the stress-strain curves computed from these data together with a friction stress, which is independent of microstructure [22]. As expected from the hybrid modelling procedure, these stress strain curves are in excellent agreement with the experimental curves from plane strain compression tests [19]. The model also uses the microstructure variables in equations (5-11) to obtain the static recrystallisation variables shown in Figure 5 [22].

Figure 6 compares the local distributions of temperature, equivalent strain, and equivalent strain rate, together with the flow stress computed by the conventional and hybrid models by linking them to finite element software for plane strain rolling. It can be seen that the temperature and strain distributions produced by both approaches are in close agreement, but there are minor systematic differences in the strain rate distributions, with the hybrid model giving a narrower band of higher strain rate. Minor differences also arise in the flow stress distributions, with the hybrid model giving slightly larger region of higher flow stress. The hybrid model also maps the distributions of microstructure variables in the deforming material assuming no static recovery in the short time after exit, as shown in Figure 7. Figure 7(c) indicates that the dislocation density in the rolled slab increases from centre to surface, but the difference for the present rolling conditions is too small to measure experimentally. The same is true for the subgrain size, Figure 7(a), but Figure 7(b) indicates that misorientation is expected to vary from 3 to more than $4^{\circ}$, which should be detectable experimentally.

Figures 8 and 9 show equivalent results from the hybrid model for the deformation variables and for the microstructure variables at the end of a PSC test. It can be seen that that the characteristic heterogeneity of the deformation variables leads to larger variations in the as-deformed microstructure variables than expected after rolling. In principle, they could all be mapped experimentally. Although measuring the distribution of dislocation density by transmission electron microscopy would be impractical, subgrain size and misorientation could be determined by electron back scattered diffraction (EBSD). However, from an industrial point of view, the consequences of these microstructure distributions on subsequent recrystallisation behaviour are of more interest than the distributions of the deformed microstructures themselves.

Experimental measurements of the distribution of fractions recrystallised after annealing for four different times are compared directly with the predictions of the conventional and hybrid models in Figure 10. For the conventional model, the distributions of temperature, equivalent strain and strain rate shown in Figure 8 were applied in equations (18) and (19). It can be seen that the overall distributions show similar characteristics, with the hybrid model results appearing to be superior, but detailed comparison is made difficult by the fact that the fractions recrystallised predicted by the models differ somewhat from the experimental value for a given annealing time. As described in Section 4, the mean fractions recrystallised in the deformation zone were determined from the distributions. The results are shown as recrystallisation curves in Figure 11. All the curves are of similar slope, and the hybrid model gives kinetics reasonably close to experiment. In fact, the 
kinetics are in surprisingly good agreement considering that the hybrid model was trained on data [13], and the constant $\alpha$ in equation (19) was found from data [13], which were not from the same research as the experimental observations [21]. In contrast, the conventional model gives a value of $t_{50}$ more than twice the experimental value. In this context, it is of interest to note that in the experimental research [21] two different alloys of similar composition were investigated and the value of $\alpha$ was found to differ by about a factor of three. The computed curves in Figure 11 cannot be accurately fitted to an Avrami equation, but are consistent in slope with an exponent of 1 found experimentally [21].

Figure 12 shows the recrystallisation curves computed for four locations in which the local equivalent strain is $0.670 \pm 0.005$. These are compared with the curve for the experimental data determined from the contours in Figure 10. It can be seen that all the data fit equation (18) with a time exponent of 2 and optimised values of $t_{50}$ for each case. These values of $t_{50}$ differ somewhat for each of the selected elements because, as shown in Figure 8 , the final strain rates and temperatures differ somewhat, leading to differences in the final value of $Z$ in equation (19). Comparison of the curves in Figure 12 for elements having a final strain of 0.67 with the curves in Figure 11 for the mean fraction recrystallised in the deformation zone for a nominal equivalent strain of 0.67 shows that the heterogeneity of deformation conditions in the deformation zone causes recrystallisation to take place over a much longer time period, but has only a relatively minor effect on the value of $t_{50}$.

From the recrystallisation curves in Figure 11, the annealing times required in the models to give the same mean fractions recrystallised in the deformation zone as for the experimental observations were determined. Figure 13 shows the distributions of recrystallised fraction predicted by the two models for mean recrystallised fractions of $0.11,0.42,0.65$, and 0.87 , which are the same as for the experimental distributions after annealing times of 40,100,160, and 320 s shown in Figure 10. From Figure 13 it is clear that the two models produce closely similar results. Comparison of these results with the experimental results in Figure 10 for the same fractions recrystallised shows that there are some systematic differences in the distributions. It is not certain why these differences arise, but it is probable that they originate from using mean fractions recrystallised in the deformation zone in deducing equation (19) used in the conventional model, and using mean fractions recrystallised correlated with nominal equivalent strain in the training data for the hybrid model, i.e. the redundant shear strains in the strain distribution, Figure $8(\mathrm{~b})$, were not considered, so the effects of all the deformation variables, and changes with strain were not captured in either model.

From these results, it is apparent that the hybrid model should be trained from experimental values of microstructure variables mapped onto the local deformation conditions computed by the FE model. As emphasised by Pietrzyk et al [24], a heterogeneous distribution of deformation conditions, as in the plane strain compression test, Figure 8, then becomes advantageous, because it provides a wide range of data from a single test. Having trained the model on such data, it should then apply accurately to the heterogeneous deformation conditions on industrial thermomechanical processing, such as rolling. In fact, by using EBSD instead of optical and transmission electron microscopy, it is feasible to extend the range of training data 
available by extending the range of heterogeneous and transient deformation conditions in the PSC tests, e.g. by testing specimens of different breadths to examine the influence of different deviations from plane strain conditions, or by testing with tools at different temperatures from the specimens to extend the range of thermal transients. Three dimensional FE models are available to analyse such results [25] and the models can handle the asymmetry sometimes found in experimental tests [26]. A wider range of training data would extend the accurate range of applicability of hybrid models for mapping microstructure variables in industrial thermomechanical processes.

\section{Conclusions}

The finite element method has been used in combination with intelligent hybrid neuro-fuzzy models for detailed modelling of flow stress for a thermomechanically processed aluminium alloy. The technique has been applied successfully to both hot rolling and PSC testing. The results are compared with those using traditional empirical equations for modelling the material properties. Both approaches have produced similar results in terms of stress, strain rate, strain and temperature, but the hybrid modelling approach gives the additional benefit of calculating the internal states of the process (dislocation density, subgrain size and misorientation) in a dynamic manner. In addition, when compared with experimental data, the recrystallisation kinetics predicted by the hybrid model were much more accurate than by conventional empirical equations.

\section{Acknowledgment}

The authors gratefully acknowledge the UK EPSRC (Engineering and Physical Sciences Research Council) for their financial support under grant number GR/R70514/01.

\section{References}

[1] Q. Zhu, H. R. Shercliff and C. M. Sellars: Int. Conf. on 'Thermomechanical Processing of Steel and other Materials', THERMEC'97, 1997, 2039-2045.

[2] Q. Zhu and C. M. Sellars: Proc. 6th Int. Conf. on 'Aluminium Alloys, their Physical and Mechanical Properties', ICAA-6, Toyohashi, Japan, July 1998.

[3] H. U. Loffler, D. Gade, R. Doll, H. Muller, T. Peuker and G. Sorgel: IFAC Workshop on 'Future Trends in Automation in Mineral and Metal Processing, Helsinki, Finland, S. L. Jämsä-Jaunela, and E. Vapaavuori (Eds), Pergamon, , 2000, 395-400.

[4] M. F. Abbod, D. A. Linkens and Q. Zhu: Proc. 3rd Int. Conf. on 'Intelligent Processing and Manufacturing of Materials', IPMM'2001, Vancouver, Canada, 29 July - 3 August, 2001.

[5] R. Colas and C. M. Sellars: Journal of Testing and Evaluation, 1987, 342-349.

[6] Q. Zhu and C. M. Sellars: Monterey Institute of Advanced Studies, T. R. McNelley (Ed), California, USA, 1996a, 195-202. 
[7] C. M. Sellars and Q. Zhu: Hot Deformation of Aluminium Alloys II, T. R. Bieler, L. A. Lalli and S. R. MacEwen, Eds., Minerals, Metals and Materials Society/AIME, Rosemont, USA, 1998, 185-198.

[8] C. M. Sellars and Q. Zhu: Risø National Laboratory, Roskilde, Denmark, J. B. Bilde-Sørensen et al, (Eds), 1999, 167-182.

[9] Q. Zhu and C. M. Sellars: Microstructural evolution of aluminium-magnesium alloys during thermomechanical processing. Materials Science Forum, 2000, 331-337, 409-425.

[10] G. T. Baxter, T. Furu, Q. Zhu, J. A. Whiteman and C. M. Sellars: Acta Materialia, 1999, 47(8), 23672376.

[11] T. Furu, H. R. Shercliff, G. J. Baxter and C. M. Sellars: Acta Materialia, 1999, 47(8), 2377-2389.

[12] Q. Zhu and C. M. Sellars: The Institute of Materials, J. H. Beynon, P. Ingham, H. Teichert and K. Waterson (Eds), London, 1996b, 158-166.

[13] C. M. Sellars and Q. Zhu: Materials Science and Engineering, 2000, A280, 1-7.

[14] M. F. Abbod, D. A. Linkens and Q. Zhu: Materials Science and Engineering A, 2002, A333, 397-408.

[15] T. Dean, J. Allen and Y. Aloimonos: "Artificial Intelligence Theory and Practice", the Benjamin/ Cummings Publishing Company, Inc, Califorina, 1995.R. Colas and C. M. Sellars: Journal of Testing and Evaluation, 1987, 342-349.

[16] J. R. Jang: IEEE transaction on Systems, Man and Cybernetics, 1993, 23(3), 665-685.

[17] G. Bersini, C. Bontempi and Decaestecker: International Conference on Artificial Neural Networks, F. Fogelman-Soulie', P. Gallinari (Eds.), Paris, 1995, 1, 169-174.

[18]E. S. Puchi, J. H. Beynon and C. M. Sellars: Int. Conf. on 'Physical Metallurgy of Thermomechanical Processing of Steels and Other Metals', THERMEC'88, Tokyo, June 1988, Iron and Steel Inst. of Japan, $572-579$.

[19] H. Shi, A. J. McLaren, C. M. Sellars, R. Shahani and R. Bolingbroke: Materials Science and Technology, 1997, 13, 210-216.

[20]E. S. Puchi Cabrera: 'Effect of the Deformation History on the Recrysta11ization Kinetics of A1-1\% Mg Alloy', PhD Thesis, University of Sheffield, Sheffield, UK, 1983.

[21] C. M. Sellars, A. M. lrisarri and E. S. Puchi: Microstructural Control in Aluminium Alloys, eds. E. Henry Chia and H. J. McQueen, Met. Soc. AIME, Warrendale, PA., 1986, 179-196.

[22] Q. Zhu, M. F. Abbod, J. Talamantes-Silva, C. M. Sellars, D. A. Linkens and J. H. Beynon: Acta Mater., 2003, 51, 5051-5062.

[23] M. F. Abbod, C. M. Sellars. D. A. Linkens, Q. Zhu and M. Mafouf: Mats. Sci. and Eng., 2005, A395, 3545. 
[24]M. Pietrzyk, Z. Kedzierski and J. G. Lenard:, Proc. Int. Conf. on 'Numerical Methods in Industrial Forming Processes', NUMIFORM'98, Enschede, Netherlands, June 1998, eds. J. Huetink and F. P. T. Baaijens, Balkema, Rotterdam, 163-168.

[25] M. S. Mirza and C. M. Sellars: Mater. Sci. and Technol., 2001, 17, 1142-1148.

[26] M. S. Mirza and C. M. Sellars: Mater. Sci. and Technol., 2007, 23, 567-576. 


\section{List of Figures}

Figure 1. Block diagram of the microstructure hybrid model

Figure 2. Schematic diagram of the hot rolling process, showing the complex thermal phenomena taking place during rolling.

Figure 3. Finite element meshes used for the modelling of (a) rolling, showing the fine elements used to give more accurate representation at the roll-stock interface (b) PSC testing, showing the quadrant of the specimen for which results are computed.

Figure 4. Hybrid modelling results for microstructure evolution during static recrystallisation of $\mathrm{Al}-1 \% \mathrm{Mg}$ on annealing at the deformation temperature after deformation at a strain rate of $1 \mathrm{~s}^{-1}$ and temperatures of 300

$-600{ }^{\circ} \mathrm{C}$ (a) dislocation density, $\rho, \mathrm{m}^{-2}$, (b) misorientation between subgrains, $\theta^{\circ}$, (c) subgrain size, $\delta$, $\mu \mathrm{m}$, and (d) the resulting equivalent stress - strain curves.

Figure 5. Hybrid modelling results for microstructure evolution during static recrystallisation of $\mathrm{Al}-1 \% \mathrm{Mg}$ on annealing at the deformation temperature after deformation at a strain rate of $1 \mathrm{~s}^{-1}$ and temperatures of 300 - $600{ }^{\circ} \mathrm{C}$ (a) nucleation density, $\mathrm{N}_{\mathrm{v}}, \mathrm{m}^{-3}$, (b) recrystallised grain size, $\mathrm{d}_{\mathrm{rex}}, \mu \mathrm{m}$, (c) time for $50 \%$ recrystallisation, $\mathrm{t}_{50}, \mathrm{~s}$.

Figure 6. Comparison of the distributions of (a) temperature, ${ }^{\circ} \mathrm{C}$, (b) equivalent strain, (c) equivalent strain rate, $\mathrm{s}^{-1}$, and (d) flow stress, MPa, calculated by finite element modelling using conventional empirical equations (left) and using the hybrid model (right) for rolling a $20 \mathrm{~mm}$ thick slab with a uniform initial temperature of $400{ }^{\circ} \mathrm{C}$ to $10 \mathrm{~mm}$ thickness using rolls of $136 \mathrm{~mm}$ diameter and a peripheral speed of $60 \mathrm{RPM}$.

Figure 7. Hybrid model predictions for evolution of the microstructural variables during rolling (a) subgrain size, $\delta, \mu \mathrm{m}$, (b) misorientation between subgrains, $\theta$, ${ }^{\circ}$, and (c) dislocation density, $\rho, \mathrm{m}^{-2}$.

Figure 8. Hybrid model predictions for the distributions of (a) temperature, ${ }^{\circ} \mathrm{C}$, (b) equivalent strain, (c) equivalent final strain rate, $\mathrm{s}^{-1}$ and (d) final flow stress, MPa, calculated by finite element modelling for PSC testing a specimen of $10 \mathrm{~mm}$ initial thickness at an initial temperature of $400^{\circ} \mathrm{C}$ and a nominal equivalent strain rate of $1 \mathrm{~s}^{-1}$ to a nominal equivalent strain of 0.67 .

Figure 9. Hybrid model predictions for the distributions of the microstructure variables after PSC testing (a) subgrain size, $\delta, \mu \mathrm{m},(\mathrm{b})$ misorientation between subgrains, $\theta^{\circ}$, (c) dislocation density, $\rho, \mathrm{m}^{-2}$. 
Figure 10. Comparison of the distributions of recrystallised fraction computed by the hybrid and conventional models with experimental results in specimens annealed at $400^{\circ} \mathrm{C}$ for (a) 40 , (b) 100 , (c) 160 , and (d) $320 \mathrm{~s}$ after PSC tests.

Figure 11. Comparison of recrystallisation curves predicted by the models and found experimentally for the mean fractions recrystallised in the deformation zone.

Figure 12. (a) Upper-left quadrant of specimen showing the positions of elements with local equivalent strains of 0.67, (b) recrystallisation curves for elements 1 to 4 (numbered from left to right in Figure 12(a)).

Figure 13. Distributions of recrystallised fractions predicted by the hybrid and conventional models for mean fractions recrystallised in the deformation zone of (a) 0.11 , (b) 0.42 , (c) 0.65 , and (d) 0.87 after PSC tests. 